\title{
Performance Evaluation of Direct-Sequence Spread Spectrum Multiple-Access for Indoor Wireless Communication in a Rician Fading Channel
}

\author{
RAMJEE PRASAD, Senior Member, IEEE, HOWARD SEWBERATH MISSER, \\ Member, IEEE, and ADRIAAN KEGEL, Member, IEEE.
}

\begin{abstract}
The bit error probability in a Rician fading channel is evaluated for indoor wireless communications considering Direct-Sequence Spread Spectrum Multiple Access (DS/SSMA) with Differential Phase Shin Keying (DPSK) modulation and two types of diversity: selection diversity and maximal ratio combining. The performance of the indoor radio system is also obtained in terms of outage probability and bandwidth efficiency. The analysis is done for a star-connected multiple access radio network. Furthermore the influence of three types of Forward Error Correcting (FEC) codes namely, the $(15,7)$ BCH code, the $(7,4)$ Hamming code and the $(23,12)$ Golay code, on the performance is studied. Computational results are presented for suitable values of Rician parameters in an indoor environment and using Gold codes as spread spectrum codes.
\end{abstract}

\section{INTRODUCTION}

Indoor Wireless communication has recently drawn the attention of many researchers $[1-14,16]$ due to its significant advantages over the conventional cabling: modiltity of users, elimination of wiring and rewiring, drastical reduction of wiring in new buildings, flexibility of changing or creating new communication services, time and cost saving, and reduction of the down time of services. Much attention is being paid to the use of Direct-Sequence Spread-Spectrum (DS/SS) modulation for indoor wireless multiple access communication, over multipath fading channels $[1-8,16]$. DS/SS modulation provides both multiple access capability and resistance to multipath fading.

In this paper, we obtain the performance of Direct

Paper approved by James S. Lehnert, the Editor for Modulation \& Signal Design of the IEEE Communications Society. Manuscript received: December 20, 1990; revised: September 25, 1992; January 7, 1993; November 15,1993 . This paper was presented in part at the First IEEE International Symposium on spread spectrum Techniques and Applications, King's College London, September 1990.

R. Prasad and A. Kegel are with the Telecommunications and TrafficControl Systems Group, Delft University of Technology, P.O. Box 5031, 2600 GA Delft, The Netherlands. H. S. Misser is with Dr. Neher Laboratories, PTT Research, P.O. Box 421, 2260 AK Leidschendam, The Netherlands.

IEEE Log Number 9410091.
Sequence Spread Spectrum Multiple Access (DS/SSMA) with DPSK, in an indoor Rician fading radio channel. The performance of a DS/SSMA system in indoor Rayleigh fading channels using DPSK or CPSK was considered in [1-4]. However, recent multipath measurements of the indoor radio channel at $800 / 900 \mathrm{MHz}$ and $1.75 \mathrm{GHz}$ characterize the indoor environment as a frequency selective, Rician fading channel $[9,10]$. Measurements in factory environments have also indicated that Rician distribution fits the experimental data [11]. A recent paper by Wang and Moeneclaey [16] has appeared on a similar topic. That paper [16] addressed the performance of Hybrid DS/SFH-SSMA systems in indoor Rician-channels using maximal ratio combining and coding. Thus, there is a small overlap between [16] and this paper as far as the performance using maximal ratio combining is concerned.

To avoid the need for synchronous carrier recovery at the receiver, which is a difficult task in a multipath fading environment, DPSK is used as modulation scheme. Also, selection diversity and maximal ratio combining are used to combat the multipath fading.

Radiowave propagation measurements showed that the maximum rms delay spread at $850 \mathrm{MHz}, 1.7 \mathrm{GHz}$ and 4.0 $\mathrm{GHz}$ did not exceed $270 \mathrm{nsec}$. in the larger buildings and 100 nsec. in the smaller buildings [12]. In this paper the performance is evaluated for the rms delay spread in the range of $50 \mathrm{nsec}$. to $250 \mathrm{nsec}$.

In the performance analysis average power control is assumed to make sure that all signals arrive at the base station with the same average power. As explained in [3] this can be accomplished as follows. The base station transmits a signal common to all users. The users monitor the average level of this signal. This information can now be used to adjust the transmitted power at the user location.

The paper is organized as follows. In section II the system model is introduced. In section III the performance is derived in terms of bit error probability, outage probability and bandwidth efficiency. Numerical results and discussions are presented in section IV. In section V the conclusions can be found. 


\section{SYSTEM MODEL}

In this section we present the transmitter, the channel and the receiver model. The transmitter and receiver models are similar to those in [3].

\section{A. Transmitter Model}

$K$ active users may simultaneously transmit to a base station using DS/SSMA with DPSK modulation. The (differentially encoded) data waveform of user $k$ is denoted as

$$
b_{k}(t)=\sum_{j=-\infty}^{\infty} b_{k}^{j} P_{T_{b}}\left(t-j T_{b}\right), b_{k}^{j} \in(1,-1)
$$

where $P_{T}$ is a rectangular pulse of unit height and duration $T$, and $T_{b}$ is the duration of one data bit. Each user has a unique spread spectrum code of $\mathbf{N}$ chips that fits into one data bit, i.e., $T_{b}=N T_{c}$ where $T_{c}$ is the chip duration. The spread spectrum code of user $k$ is

$$
a_{k}(t)=\sum_{i} a_{k}^{i} P_{T_{c}}\left(t-i T_{c}\right), a_{k}^{i} \in(1,-1)
$$

where $i=. .,-1,0,1, \ldots$ and $\mathrm{a}_{\mathrm{k}}^{\mathrm{i}}=\mathrm{a}_{\mathrm{k}}^{\mathrm{i}+\mathrm{N}}$.

Now the signal at the output of the $k$ th transmitter can be written as

$$
S_{k}(t)=A a_{k}(t) b_{k}(t) \cos \left(\omega_{c} t+\theta_{k}\right)
$$

where $A$ is the amplitude of the carrier, $\omega_{c}$ is the common angular carrier frequency, and $\theta_{k}$ is the carrier phase for the $k$ th user.

\section{B. Channel model}

We assume that the signal bandwidth is much larger than the coherence bandwidth of the radio channel which assures us of the existence of multiple resolvable paths. The (complex) lowpass equivalent impulse response of the bandpass channel for the link between the $k$ th user and the base station is written as

$$
h_{k}(t)=\sum_{l=1}^{L} \beta_{l k} \delta\left(t-\tau_{l k}\right) \exp \left(j \gamma_{l k}\right)
$$

Here $\beta$ is the path gain, $\tau$ is the path delay, $\gamma$ is the path phase, and $\mathrm{L}$ is the number of resolvable paths. The index $l k$ refers to the $l$ th path of the $k$ th user, and $\mathrm{j}=\sqrt{ }-1$. The number of paths may be either fixed or randomly changing. Here fixed values for $L$ are assumed. The number of paths is upper bounded by [4]

$$
L=\left\lfloor\frac{T_{m}}{T_{c}}\right\rfloor+1
$$

where $T_{m}$ is the rms delay spread and $T_{c}$ is the duration of a code chip.

We assume that the path phases on arrival at the receiver, $\left(\omega_{\mathrm{c}} \tau_{l \mathrm{k}}+\gamma_{\mathrm{lk}}\right)$, are independently uniformly distributed over $[0,2 \pi]$. We also assume that the path delays are independently uniformly distributed over $\left[0, T_{b}\right]$. Unlike $[1-4]$, where $\beta_{\mathrm{lk}}$ was assumed to be Rayleigh distributed, we shall assume that the path gains are independent Rician distributed random variables. This is in accordance with recent measurements done in office $[9,10]$ and factory $[11]$ buildings.

The Rician probability density function (PDF) is given as

$$
\begin{aligned}
& p_{\beta}(r)=\frac{r}{\sigma^{2}} \exp \left(-\frac{r^{2}+S^{2}}{2 \sigma^{2}}\right) I_{0}\left(\frac{S r}{\sigma^{2}}\right) \\
& 0 \leq r \leq \infty, S \geq 0 \\
& R=\frac{S^{2}}{2 \sigma^{2}}
\end{aligned}
$$

where $I_{o}()$ is the modified Bessel function of the first kind and zero order, $S$ is the peak value of the diffuse radio signal due to the superposition of the dominant (line-ofsight) signal and the time invariant scattered signals reflected from walls, ceiling and stationary inventory, $\sigma^{2}$ is the average signal power that is received over specular paths.

From [10] we know that typical values for $\mathrm{R}$ are $6.8 \mathrm{~dB}$ and $11 \mathrm{~dB} . \mathrm{R}=6.8 \mathrm{~dB}$ corresponds to a 30 -year-old brick building with reinforced concrete and plaster and $\mathrm{R}=11 \mathrm{~dB}$ corresponds to a building having the same construction, but with an open-office interior floor plan, and non-metallic ceiling tiles throughout.

\section{Receiver model}

Using equation (3) and (4) the received signal can be written as

$$
\begin{aligned}
r(t)=\sum_{k=1}^{K} \sum_{l=1}^{L} A \beta_{l k} a_{k}\left(t-\tau_{l k}\right) b_{k}\left(t-\tau_{l k}\right) \\
\cdot \cos \left(\omega_{c t}+\phi_{l k}\right)+n(t) \\
\phi_{l k}=\omega_{c} \tau_{l k}+\gamma_{l k}
\end{aligned}
$$

where $n(t)$ is the white Gaussian noise with two-sided power 
spectral density $\mathrm{N}_{\mathrm{o}} / 2$. The receiver model consists of a matched filter, a DPSK demodulator and diversity processing components, as shown in figure 1.

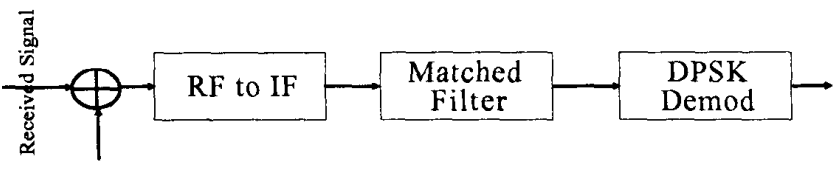

White Gaussian Noise

Fig. 1. Block diagram of the spread spectrum receiver using DPSK modulation.

As in [3] equation (7) can be divided into two parts: inphase and a quadrature component. Selecting user 1 as the reference user, the output (in-phase and quadrature component) of the matched filter of user 1 at the sampling point $\left(t=T_{b}\right)$ is given as

$$
\begin{aligned}
g_{x}\left(T_{b}\right)= & \sum_{k=1}^{K} \sum_{l=1}^{L} A \beta_{l k} \cos \left(\phi_{l k}\right)\left[b_{k}^{-1} R_{1 k}\left(\tau_{l k}\right)\right. \\
& \left.+b_{k}^{0} \hat{R}_{1 k}\left(\tau_{l k}\right)\right]+\eta \\
g_{y}\left(T_{b}\right)= & \sum_{k=1}^{K} \sum_{l=1}^{L} A \beta_{l k} \sin \left(\phi_{l k}\right)\left[b_{k}^{-1} R_{1 k}\left(\tau_{l k}\right)\right. \\
& \left.+b_{k}^{0} \hat{R}_{1 k}\left(\tau_{l k}\right)\right]+v
\end{aligned}
$$

where $g_{x}$ and $g_{y}$ are the in-phase and the quadrature component, respectively, $b_{k}{ }^{-1}$ and $b_{k}^{0}$ are the previous and current data bit, respectively, and

$$
\begin{aligned}
& R_{1 k}(\tau)=\int_{0}^{\tau} a_{k}(t-\tau) a_{1}(t) d t \\
& \hat{R}_{l k}(\tau)=\int_{\tau}^{T_{b}} a_{k}(t-\tau) a_{1}(t) d t
\end{aligned}
$$

The noise samples $\eta$ and $v$ are independent, zero-mean Gaussian random variables with identical variance $\sigma_{n}{ }^{2}=$ $\mathrm{N}_{\mathrm{o}} \mathbf{T}_{\mathrm{b}}$.

Let us assume without loss of generality that the receiver synchronizes to the $j$ th path of user 1 , so that $\tau_{j 1}=0$ and $\phi_{j 1}$ $=0$ [3]. The complex envelope of the signal at the current sampling instant then is

$$
\begin{aligned}
z_{0}= & A \beta_{j 1} T_{b} b_{1}^{0}+\sum_{k=1}^{K} A\left(b_{k}^{-1} X_{k}+b_{k}^{0} \hat{X}_{k}\right) \\
& +j \sum_{k=1}^{K} A\left(b_{k}^{-1} Y_{k}+b_{k}^{0} \hat{Y}_{k}\right)+\left(\eta_{1}+j v_{1}\right)
\end{aligned}
$$

where

$$
\begin{gathered}
X_{1}=\sum_{\substack{l=1 \\
l \neq j}}^{L} R_{11}\left(\tau_{l l}\right) \beta_{l 1} \cos \left(\phi_{l I}\right) ; \\
\hat{X}_{1}=\sum_{\substack{l=1 \\
l \neq j}}^{L} \hat{R}_{11}\left(\tau_{l l}\right) \beta_{l l} \cos \left(\phi_{l l}\right) \\
Y_{1}=\sum_{\substack{l=1 \\
l \neq j}}^{L} R_{11}\left(\tau_{l 1}\right) \beta_{l 1} \sin \left(\phi_{l 1}\right) ; \\
\hat{Y}_{1}=\sum_{l=1}^{L} \hat{R}_{11}\left(\tau_{l 1}\right) \beta_{l 1} \sin \left(\phi_{l 1}\right)
\end{gathered}
$$$$
l \neq j
$$

and

$$
\begin{gathered}
X_{k \neq 1}=\sum_{l=1}^{L} R_{1 k}\left(\tau_{l k}\right) \beta_{l k} \cos \left(\phi_{l k}\right) \\
\hat{X}_{k}=\sum_{\substack{k \neq 1 \\
l=1}}^{L} \hat{R}_{1 l}\left(\tau_{l k}\right) \beta_{l k} \cos \left(\phi_{l k}\right) \\
Y_{k \neq 1}=\sum_{l=1}^{L} R_{1 k}\left(\tau_{l k}\right) \beta_{l k} \sin \left(\phi_{l k}\right) ; \\
\hat{Y}_{k}=\sum_{l=1}^{L} \hat{R}_{1 k}\left(\tau_{l k}\right) \beta_{l k} \sin \left(\phi_{l k}\right)
\end{gathered}
$$

Involving only $\mathrm{b}_{\mathrm{k}}{ }^{-1}$ and $\mathrm{b}_{\mathrm{k}}{ }^{0}$ means that it is assumed that $\tau_{\mathrm{jk}}$ $\geq 0$. This assumption can be made without loss of generality for two reasons: 1 ) all bits, except $b_{1}{ }^{-1}$ and $b_{1}{ }^{0}$, are 1 or -1 with equal probability; 2 ) it is not important for the crosscorrelation of two spread spectrum codes what the sign of the phase difference between the codes is.

DPSK demodulation is now achieved by taking the real part of $z_{0} z_{-1}^{*}$, where $z^{*}$ denotes complex conjugate of $z$.

\section{Performance ANAIYSIS}

In this section the bit error probability and the outage probability for both selection diversity and maximal ratio combining are derived. The bit error probability with FEC coding and the bandwidth efficiency are also obtained.

\section{A. Selection diversity}

1) Bit error probability: The selection diversity scheme is based on selecting the strongest of $L$ resolvable paths. By using multiple antennas the highest possible order of diversity, i.e. number of paths to choose from, can be increased to $M_{\max }=\mathrm{kL}$ where $\mathrm{M}_{\max }$ is the maximum order 
of diversity and $\mathrm{k}$ is the number of antennas. To derive the PDF of the strongest path $\left(\beta_{\max }\right)$, it is essential to note that the cumulative density function (CDF) of $\beta_{\max }$ is just the $\mathrm{CDF}$ of the PDF in equation (6) raised to the power of the order of diversity, $M$, hence

$$
\begin{gathered}
p_{\beta_{\max }}(r)=M\left[1-Q\left(\frac{s}{\sigma}, \frac{r}{\sigma}\right)\right]^{M-1} \frac{r}{\sigma^{2}} \\
\cdot \exp \left(-\frac{r^{2}+s^{2}}{2 \sigma^{2}}\right) I_{o}\left(\frac{s r}{\sigma^{2}}\right)
\end{gathered}
$$

where $Q(a, b)$ is the Marcum Q-function. Designate the decision variable for DPSK modulation $\xi=\operatorname{Re}\left[\mathrm{z}_{0} z_{-1}^{*}\right]$, where $\operatorname{Re}[a]$ denotes the real part of $a$. The decision variable obtained from demodulation of the strongest path is written as $\xi_{\max }$. Now the bit error probability in the case of selection diversity is defined as

$$
\begin{gathered}
P_{e} \Delta P\left(\xi_{\max }<0 \mid b_{1}^{0} b_{1}^{-1}=1\right) \\
\Delta P\left(\xi_{\max }>0 \mid b_{1}^{0} b_{1}^{-1}=-1\right)
\end{gathered}
$$

In the analysis it is assumed that $b_{1}{ }^{0} b_{1}{ }^{-1}=1$, i.e. $b_{1}{ }^{0}=$ $b_{1}^{-1}=1$.

If we assume that all path delays are given and $\beta_{\max }$ is correctly selected, the bit error probability can be obtained using equation (7A.26) of [15]

$$
\begin{gathered}
P_{e} \mid \beta_{\text {max }},\left\{\tau_{l k}\right\}=Q(a, b)-\frac{1}{2}\left[1+\frac{\mu}{\sqrt{\mu_{0} \mu_{-1}}}\right] \\
\cdot I_{o}(a b) \exp \left(-\frac{a^{2}+b^{2}}{2}\right)
\end{gathered}
$$

Here $a, b, \mu, \mu_{0}$ and $\mu_{-1}$ are defined as $\left(b_{1}{ }^{0}=b_{1}{ }^{-1}=1\right)$

$$
\begin{gathered}
a \Delta \frac{m}{\sqrt{2}}\left(\frac{1}{\sqrt{\mu_{0}}}-\frac{1}{\sqrt{\mu_{-1}}}\right), b \Delta \frac{m}{\sqrt{2}}\left(\frac{1}{\sqrt{\mu_{0}}}+\frac{1}{\sqrt{\mu_{-1}}}\right), \\
m \Delta E\left[z_{0} \mid \beta_{\max }, b_{1}^{0}\right]=E\left[z_{-1} \mid \beta_{\max }, b_{1}^{-1}\right] \\
\mu_{0} \Delta \operatorname{var}\left(z_{0} \mid\left\{\tau_{l k}\right\}\right), \mu_{-1} \Delta \operatorname{var}\left(z_{-1} \mid\left\{\tau_{l k}\right\}\right), \\
\mu \Delta E\left[\left(z_{0}-m\right)\left(z_{-1}-m\right)^{*} \mid\left\{\tau_{l k}\right\}\right]
\end{gathered}
$$

with $E[]$ denoting statistical average and var() denoting variance.

Using equation (10) and the assumption that $b_{1}^{0}=b_{1}^{-1}$ $=1, \mathbf{M}, \mu_{0}, \mu_{-1}$ and $\mu$ are obtained as

$$
\begin{aligned}
& m= A \beta_{\max } T_{b} b_{1}^{0}=A \beta_{\max } T_{b} b_{1}^{-1} \\
& \mu_{0}=A^{2} \sum_{k=1}^{K} E\left[X_{k}^{2}+\hat{X}_{k}^{2}+Y_{k}^{2}+\hat{Y}_{k}^{2} \mid\left\{\tau_{l k}\right\}\right] \\
&+
\end{aligned}
$$

Note that all path gains involved in $\mu_{0}, \mu_{-1}$ and $\mu$ have a Rician distribution as given in Equation (6) except for the path gains associated to the paths of user 1 . The path gains of user 1 (except for the strongest path $\beta_{\max }$ ) have a conditional Rician PDF where the conditioning is on $\beta_{\max }$. The PDF becomes zero for path gains larger than $\beta_{\max }$. The distribution of $\beta_{\max }$ is given in Equation (13).

As can be seen from equation (17), the only difference between $\mu_{0}$ and $\mu_{-1}$ is constituted by the second term of $\mu_{0}$. If the number of simultaneously transmitting users, $K$, is large the contribution of this term to $\mu_{0}$ becomes relatively small $[2,3]$. If we drop this term from $\mu_{0}, a$ in equation (16) becomes zero. Equation (15) can then be simplified to

$$
\begin{gathered}
P_{e} \mid \beta_{\max },\left\{\tau_{l k}\right\}=Q(0, b)-\frac{1}{2}\left[1+\frac{\mu}{\sqrt{\mu_{0} \mu_{-1}}}\right] I_{0}(0) \\
\exp \left(-\frac{b^{2}}{2}\right)=\frac{1}{2}\left[1-\frac{\mu}{\mu_{0}}\right] \exp \left(-\frac{m^{2}}{\mu_{0}}\right)
\end{gathered}
$$

since $I_{0}(0)=1$ and $Q(0, b)=\exp \left(-b^{2} / 2\right)$. Now to remove the conditioning on $\tau_{1 \mathrm{k}}$, we approximate $\mu$ and $\mu_{0}$ by Gaussian variables and integrate equation (18) over $\mu$ and $\mu_{0}$. Likewise the conditioning on $\beta_{\max }$ is removed. Thus equation (18) reduces to

$$
\begin{aligned}
& P_{e}=\int_{-\infty}^{\infty} \int_{0}^{\infty} \frac{1}{2}\left[1-\bar{\mu} \frac{\bar{\mu}}{\mu_{0}}\right] \exp \left(-\frac{A^{2} \beta_{\max }^{2} T_{b}^{2}}{\mu_{0}}\right) \\
& \cdot p_{\beta_{\max }} p_{\mu_{0}} d \beta_{\max } d \mu_{0}
\end{aligned}
$$

For the calculation of the variance of $\mu$ and $\mu_{0}$ a specific set of Gold codes are used. The partial cross-correlation functions are calculated using the specific Gold codes and averaging over the (uniform) path delay distributions. For calculating the moments of $\mu$ and $\mu_{0}$, these calculations have to be done for all resolvable paths of all users. 
2) Outage probability: Outage probability is defined as the probability that the instantaneous bit error probability exceeds a preset threshold. We denote the threshold value as ber ${ }_{o}$. The instantaneous value of the bit error probability can be obtained using equation (19). The averaging over $\beta_{\max }$ should then be removed and a fixed value for $\beta_{\max }, \beta$, should be substituted. Equation (19) then transforms to

$$
P_{e}(\beta)=\int_{-\infty}^{\infty} \frac{1}{2}\left[1-\frac{\bar{\mu}}{\mu_{0}}\right] \exp \left(-\frac{A^{2} \beta^{2} T_{b}^{2}}{\mu_{o}}\right) p_{\mu_{0}}\left(\mu_{0}\right) d \mu_{0}
$$

The outage probality in the case of selection diversity can then be calculated as follows

$$
\begin{aligned}
P_{\text {out }} & =P\left(0 \leq \beta_{\max } \leq \beta_{0}\right)=P\left(\operatorname{ber}(\beta) \geq \text { ber }_{o}\right) \\
& =\int_{0}^{\beta_{0}} M\left[1-Q\left(\frac{s}{\sigma}, \frac{v}{\sigma}\right)\right]^{M-1} \frac{v}{\sigma^{2}} \\
& \exp \left(-\frac{v^{2}+s^{2}}{2 \sigma^{2}}\right) I_{0}\left(\frac{s v}{\sigma^{2}}\right) d v
\end{aligned}
$$

Here $\beta_{0}$ is the value of $\beta$ at which the instantaneous bit error probability is equal to ber $_{o}$. The integrated function is just the PDF of $\beta_{\max }$ where $M$ is the order of diversity. For a given value of $\beta_{0}$, the outage probability decreases as the order of diversity increases. Also a higher signal-to-noise ratio results in a lower outage probability because for a given ber $_{o}$, the value of $\beta_{\mathrm{o}}$ obtained from (20) decreases as the signal to noise ratio increases.

\section{B. Maximal ratio combining}

1) Bit error probability: With Maximal-Ratio Combining (MRC) of order $M$ the decision variable is the weighted sum of the demodulation results of $M$ copies of the signal. The weights are taken equal to the corresponding complex-valued (conjugate) channel gain $\beta_{i} \exp \left(-j \gamma_{i}\right)$. The effect of this multiplication is to compensate for the phase shift in the channel and to weight the signal by a factor that is proportional to the signal strength. A reason for using DPSK, as in our case, is that the time variations in the channel parameters are sufficiently fast to preclude the implementation of synchronous carrier recovery schemes. However, the channel variations must on the other hand be sufficiently slow so that the channel phase shifts $\left(\gamma_{\mathrm{i}}\right)$ do not change appreciably over two consecutive signalling intervals (DPSK detection). We assume that the channel parameters $\left\{\beta_{\mathrm{i}} \exp \left(-\mathrm{j} \gamma_{\mathrm{i}}\right)\right\}$ remain constant over two succesive signalling intervals. Under that condition we do not need estimates of the channel parameters because the signals are automaticaly weighted. The decision variable in the case of MRC/DPSK is

$$
\xi=\operatorname{Re}\left[\sum_{i=1}^{M}\left(A T_{b} \beta_{i} b_{1}^{0}+N_{1 i}\right)\left(A T_{b} \beta_{i} b_{1}^{-1}+N_{2 i}\right)^{*}\right]
$$

Here $N_{1 i}$ and $N_{2 i}$ are Gaussian random variables. If we assume, just as in the case of selection diversity, the multiuser interference to be Gaussian, $N_{1 i}$ and $N_{2 i}$ are the sum of the Gaussian noise power and the multi-user interference power. The multi-user interference is calculated in the same way as for selection diversity. Equation (22) is just the sum of the demodulated signals of $M$ paths. This method is therefore sometimes called predetection combining or postdemodulation combining. We will now make two assumptions. First, it can be assumed that $\mathrm{N}_{1 \mathrm{i}}$ and $\mathrm{N}_{2 \mathrm{i}}$ are independent. We have learned from the case of selection diversity that $\mu$, which is defined in Equation (16), is nearly zero. This implies that the $\operatorname{cov}\left[\mathrm{N}_{1 \mathrm{i}}, \mathrm{N}_{2 \mathrm{i}}{ }^{*}\right]$ is negligible compared with $\operatorname{var}\left[\mathrm{N}_{1 \mathrm{i}}\right]$ and $\operatorname{var}\left[\mathrm{N}_{2 \mathrm{i}}\right]$, and therefore the above assumption is reasonable [3]. Secondly, we assume that the pairs $\left(\mathrm{N}_{1 \mathrm{i}}, \mathrm{N}_{2 \mathrm{j}}\right)$ and $\left(\mathrm{N}_{1 \mathrm{j}}, \mathrm{N}_{2 \mathrm{j}}\right)$ are independent for $\mathrm{i}$ $\neq \mathrm{j}$. Mathematically this is not correct because the delays $\left\{\tau_{\mathrm{lk}}\right\}_{\mathrm{i}}$ are not independent of $\left\{\tau_{\mathrm{lk}}\right\}_{\mathrm{j}}$. However, since each set of delays is taken with reference to a different time origin (corresponding to the arrival time of the signal on the corresponding combined path), and also considering that any two resolved paths are separated by at least a chip time period, the assumption is physically reasonable [3]. With these assumptions, the bit error probability can be obtained using equations (7.4.13) and (1.1.115) of [15]

$$
P_{e}=\int_{0}^{\infty} P_{2}\left(\gamma_{b}\right) p\left(\gamma_{b}\right) d \gamma_{b}
$$

with

$$
\begin{aligned}
& P_{2}\left(\gamma_{b}\right)=\frac{1}{2^{2 M-1}} \exp \left(-\gamma_{b}\right) \sum_{n=0}^{M-1} p_{k} \gamma_{b}^{k} ; \\
& p_{k}=\frac{1}{k !} \sum_{n=0}^{M-1-k}\left(\begin{array}{c}
2 M-1 \\
n
\end{array}\right) ; \gamma_{b}=\frac{E_{b}}{N} \sum_{k=1}^{M} \beta_{k}^{2} \\
& p\left(\gamma_{b}\right)=\frac{1}{2 \sigma^{2} \frac{E_{b}}{N}}\left[\frac{\gamma_{b}}{\frac{E_{b}}{N} s_{M}^{2}}\right]^{\frac{M-1}{2}} \\
& \cdot \exp \left(-\frac{s_{M}^{2}+\gamma_{b} \frac{N}{E_{b}}}{2 \sigma^{2}}\right) I_{M-1}\left(\frac{\sqrt{\gamma_{b} \frac{N}{E_{b}}} S_{M}}{\sigma^{2}}\right)
\end{aligned}
$$

where $E_{b}=A^{2} T_{b} / 2, \beta_{k}$ is the path gain of the $k t h$ combined path, $N$ is the sum of the Gaussian noise and the multi-user 
interference which is assumed to be Gaussian, $I_{\alpha}(x)$ is the $\alpha$ th-order modified Bessel function of the first kind and $\mathrm{s}_{\mathrm{M}}{ }^{2}$ $=\mathrm{Ms}^{2}, \gamma_{\mathrm{b}}$ is the sum of the signal-to-noise ratios of the $\mathrm{M}$ combined paths, and $\mathrm{P} 2$ is the bit error probability in the case of multichannel reception over time invariant paths, i.e., the $\beta_{\mathrm{k}}$ 's are equal constants, with MRC/DPSK. Therefore, $P_{2}\left(\gamma_{b}\right)$ can be considered a conditional error probability. $\mathrm{p}\left(\gamma_{\mathrm{b}}\right)$ is the PDF of $\gamma_{\mathrm{b}}$ in the case of a Rician fading channel.

2) Outage probability: The outage probability is then obtained as

$$
P_{\text {out }}=\int_{0}^{\gamma_{b_{o}}} p\left(\gamma_{b}\right) d \gamma_{b} ; P_{2}\left(\gamma_{b_{o}}\right)=b e r_{o}
$$

where $b_{e r}$ is the preset bit error threshold.

\section{Bit error probability with FEC coding}

The specific codes considered are the $(15,7) \mathrm{BCH}$ code, the $(7,4)$ Hamming code and the $(23,12)$ Golay code. $(n, k)$ means that $\mathrm{k}$ bits are transformed to a block of $\mathbf{n}$ bits by coding. From coding theory we know that a code with hamming distance $d_{\min }$ can correct at least $t=\left(d_{\min }-1\right) / 2$ errors [15]. For the $B C H$ code $d_{\min }=5$, for the Hamming code $d_{\min }=3$ and for the Golay code $d_{\min }=7$, which means that they can correct two, one and three errors respectively. The probability of having $m$ errors in a block of $\mathbf{n}$ bits is

$$
P(m, n)=\left(\begin{array}{c}
n \\
m
\end{array}\right) P_{e}^{m}\left(1-P_{e}\right)^{n-m}
$$

Now the probability of having more than $t$ errors in a code block of $\mathrm{n}$ bits is

$$
P_{e c}=\sum_{m=t+1}^{n}\left(\begin{array}{c}
n \\
m
\end{array}\right) P_{e}^{m}\left(1-P_{e}\right)^{n-m}
$$

An approximation for the bit error probability after decoding is given in [2] as

$$
P_{e c 1}=\frac{1}{n} \sum_{m=t+1}^{n} m\left(\begin{array}{c}
n \\
m
\end{array}\right) P_{e}^{m}\left(1-P_{e}\right)^{n-m}
$$

Since the block codes can correct at least $t$ errors, equations (27) and (28) are actually upperbounds on the block error and bit error probability, respectively. Suppose we place a sphere of radius $t$ around each of the possible transmitted code words in the code space. Codes where all these spheres are disjoint and where every received code word falls in one of these spheres, are called perfect codes. They can correct $t=\left(d_{\min }-1\right) / 2$ errors. For these codes equation (28) is not an upperbound but the exact block error probability. The $(7,4)$ Hamming code and the $(23,12)$ Golay code are examples of these codes [15]. Codes where all the spheres of radius $t$ are disjoint and where every received code word is at most at distance $t+1$ from one of the possible transmitted code words, are called quasi perfect codes. They can sometimes correct $t+1$ errors. The bit error probability can be derived, using the block error probability that is given in [15], as

$$
\begin{aligned}
P_{e c 2}= & \frac{1}{n} \sum_{m=t+2}^{n} m P(m, n) \\
& +\frac{t+1}{n}\left[\left(\begin{array}{c}
n \\
t+1
\end{array}\right)-\beta_{t+1}\right] P_{e}^{t+1}\left(1-P_{e}\right)^{n-t-1} \\
\beta_{t+1}= & 2^{n-k}-\sum_{i=0}^{t}\left(\begin{array}{c}
n \\
i
\end{array}\right)
\end{aligned}
$$

Like perfect codes, quasi perfect codes are optimum on the binary symmetric channel in the sense that they result in a minimum error probability among all codes having the same block length and the same number of information bits. Therefore $P_{e c 2}$ is a lowerbound for all non perfect linear block codes. Since the $(15,7) \mathrm{BCH}$ is neither a perfect code nor a quasi perfect code, $P_{\mathrm{ec} 1}$ and $\mathrm{P}_{\mathrm{ec} 2}$ are respectively, an upperbound and an lowerbound for the bit error probability with the $(15,7) \mathrm{BCH}$ coding.

In equations (26)-(29) the channel bit error probability, $P_{e}$, is used. If the data rate, the rms delay spread and the spread spectrum code length are given, then the number of resolvable paths, $L$, can be calculated using equation (5). Note however that if the data rate is fixed, the signalling rate should be increased if FEC codes are used. This decreases the chip duration, $T_{c}$, which causes an increase in $L$. Since a higher value of $L$ means more multi-user interference, the channel bit error probability is higher if FEC codes are used. The FEC code can therefore only be useful if it is able to decrease this increased channel error probability to a value that is smaller than the value of the channel error probability without FEC coding. If the channel is very noisy, due to thermal noise or multi-user interference, FEC codes might worsen the performance. This happens when the channel error probability becomes so large, that the probability of having more errors in a code block than can be corrected becomes too large.

In order to make a fair comparison between the performance for a system with and without FEC coding it is necessary to have equal transmitted power in both cases. Since for FEC coding more bits should be transmitted, the channel SNR will be lower in that case. To be more precise, the SNR in the case of FEC coding is $\mathrm{k} / \mathrm{n}$ times the SNR in 
the case of no FEC coding.

\section{D, Bandwidth efficiency}

If the number of simultaneous users is fixed, the performance of a DS/SSMA system can be improved by using diversity, FEC codes or longer spread spectrum codes with lower crosscorrelations. In most of the cases the improvement in performance is paid for by an increase in the bandwidth. Considering this aspect, another measure of performance, bandwidth efficiency, is introduced. The bandwidth efficiency is defined as

$$
B E \Delta \frac{K_{\max } r_{b}}{W}=\frac{K_{\max } r_{c}}{N}(\text { bits } / H z)
$$

where $\mathrm{K}_{\max }$ is the maximum number of simultaneous users for which the bit error probability is less than a preset value ber $_{b e}, \mathrm{r}_{\mathrm{b}}$ is the data rate, $\mathrm{N}$ is the spread spectrum code length, $r_{c}$ is the code rate of the FEC code used and $W=$ $\mathrm{Nr}_{\mathrm{b}} / \mathrm{r}_{\mathrm{c}}$ is the total bandwidth. The code rate of a (n,k) FEC code is defined as $r_{c}=k / n$. If no FEC code is used, then $\mathbf{r}_{\mathrm{c}}=1$.
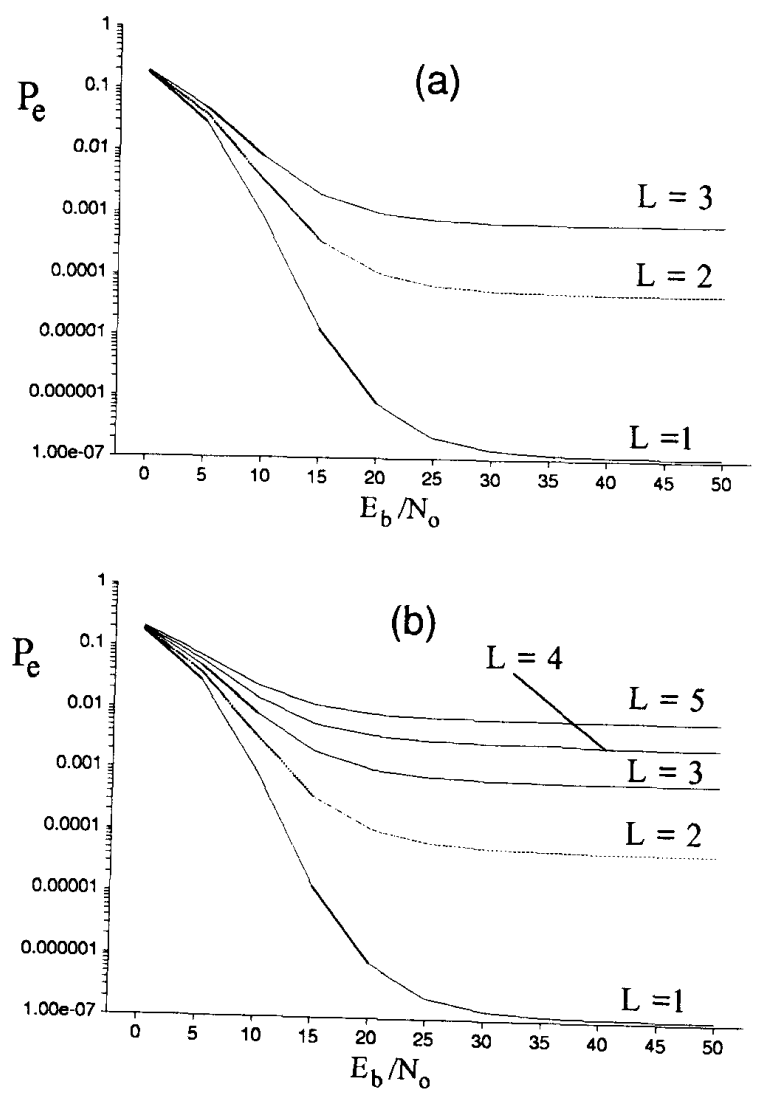

Fig. 2. The effect of the delay spread on the bit error probability for $R=$ $6.8 \mathrm{~dB}, \mathrm{M}=8$ (selection diversity), $\mathrm{N}=127, \mathrm{~K}=15$ and $(\mathrm{a}) \mathrm{r}_{\mathrm{b}}=64$ $\mathrm{kbit} / \mathrm{s}$ and $(\mathrm{b}) \mathrm{r}_{\mathrm{b}}=144 \mathrm{kbit} / \mathrm{s}$.

\section{COMPUTATIONAL RESUltS}

In this section we present results obtained from numerical evaluation of bit error probability, outage probability and bandwidth efficiency, for both types of diversity. All results were obtained using Gold codes.

1) Bit error probability with diversity: The bit error probability is evaluated as a function of the signal-to-noise ratio $E_{b} / N_{o}$. We assume that $\omega_{c} T_{b}=2 \pi l$, where 1 is an integer. This means that the transmitted power per bit is $E_{b}$ $=A^{2} T_{b} / 2$. We consider code lenghts of $N=127$ chips and of $\mathrm{N}=255$ chips. If not specified otherwise the number of simultaneous transmitting users is $K=15$. First we consider the effect of the delay spread on the performance.

In Figure 2 the effect of the delay spread on the performance is shown for selection diversity with $\mathrm{N}=127$ and for three different bit rates $\left(\mathrm{r}_{\mathrm{b}}\right)$. An important conclusion drawn from Figure 2 is that degradation in the performance only occurs if an increased value of the data rate or delay spread causes the number of paths $L$ to increase. This happens because an increase in $\mathrm{L}$ increases the multi-user interference.

Figure 3 depicts the effect of the order of diversity in the case of selection diversity. It is confirmed that diversity can significantly improve the performance.

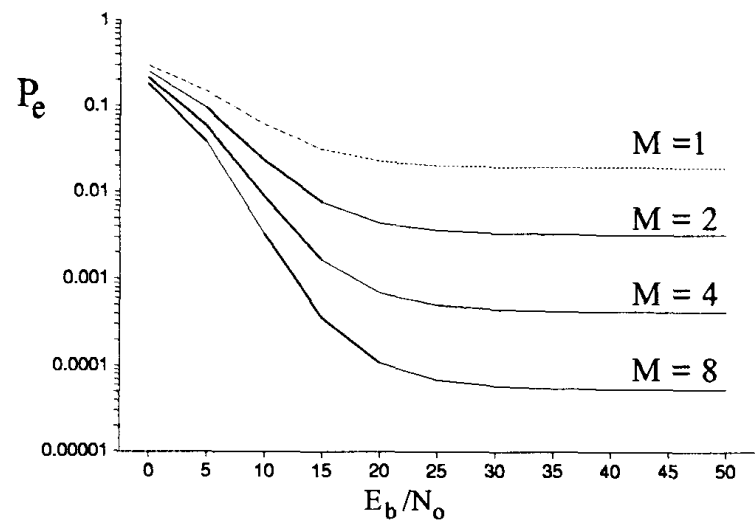

Fig. 3. The effect of the order of diversity on the bit error probability for selection diversity with $R=6.8 \mathrm{~dB}, \mathrm{~L}=2$ and $\mathrm{N}=127$.

Figure 4 shows the effect of $R$ on the performance for selection diversity with $M=4$ and $L=2$. An increase in the Rician parameter $R$ results in: 1) a better wanted signal, and 2) a worse (stronger) interference signal, i.e., more interference noise.

It is seen that for sufficiently high SNR (in this case for an SNR higher than $16 \mathrm{~dB}$ ) this results in a better performance for $R=11$ as compared to $R=6.8$. For lower SNR the increased interference noise in combination with the thermal noise plays a dominant role, which in this case causes the performance to deteriorate. 


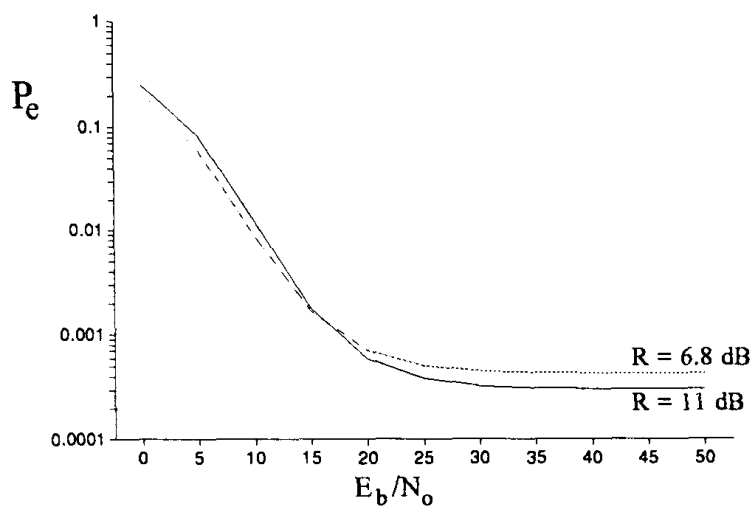

Fig. 4. The influence of the Rician parameter $\mathbf{R}$ on the bit error probability for selection diversity with $M=4, L=2$ and $N=127$.

We now compare the performance for $\mathrm{N}=127$ and $\mathrm{N}=$ 255. Let $T_{m}=185 \mathrm{~ns}$ and $r_{b}=64 \mathrm{kbit} / \mathrm{s}$. If $\mathrm{N}=127$ then $\mathrm{L}$ $=2$ and if $N=255$ then $L=4$. As opposed to Figure 2, performance is enhanced by an increase in the value of $\mathrm{L}$. This happens because the Gold codes of length 255 have better (lower) cross-correlations. Thus, the decrease in interference power per interference signal is such that even though the number of interfering signals $(\mathrm{KL}-1)$ increases, the total amount of interference power decreases. Therefore, it is seen from Figure 5 that at the cost of a doubled bandwidth, codes of 255 chips improve the performance.

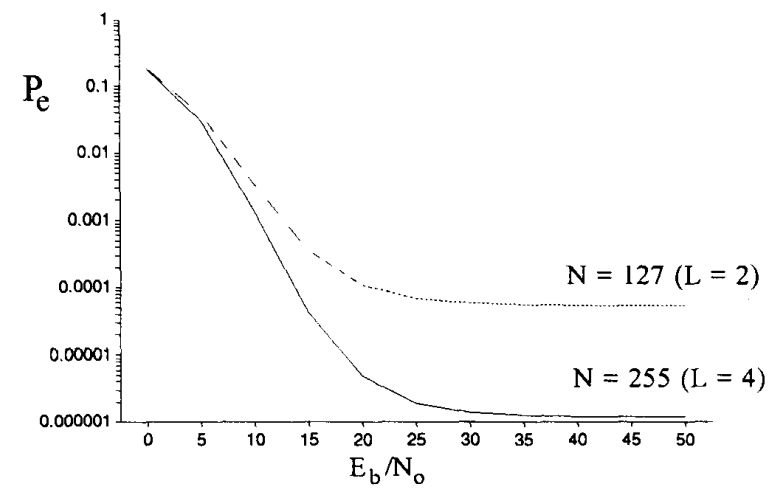

Fig. 5. Comparison of the bit error probability of $N=127$ and $N=255$ for selection diversity with $M=8, r_{b}=64 \mathrm{kbit} / \mathrm{s}, T_{m}=185 \mathrm{~ns}$ and $\mathrm{R}=$ $6.8 \mathrm{~dB}$.

Because in the case of Rician fading there is a dominant (line-of-sight) signal component, we expect the performance to be better than in the case of Rayleigh fading. Comparing the results of [3] for the Rayleigh fading case with our results, confirms this.

In Figure 6 the performance of selection diversity is compared to the performance of maximal ratio combining. As expected, maximal ratio combining yields significantly better performance than selection diversity except for very low SNR. This especially holds for higher orders of diversity.

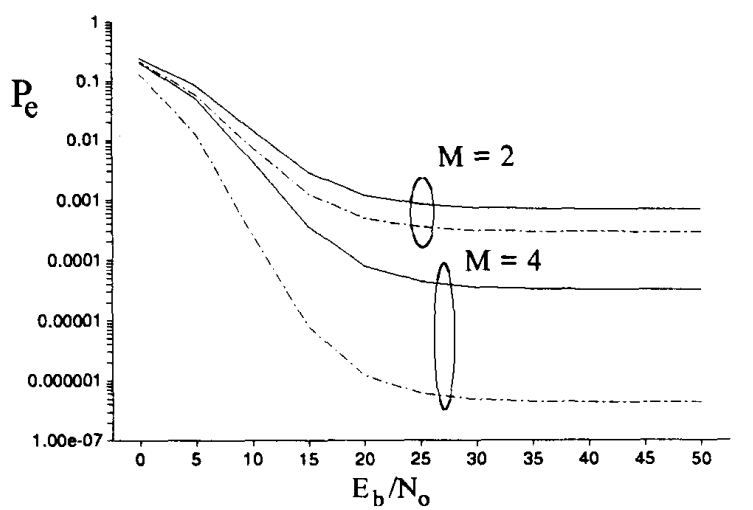

Fig. 6. Comparison of the bit error probability with selection diversity $\left(-\frac{}{2}\right)$ and maximal ratio combining $(--)$ for $R=6.8 \mathrm{~dB}, \mathrm{M}=2,4$ and $\mathrm{N}=255$.

The accuracy of the calculation method is illustrated in Figure 7. The plot compares results obtained analytically with results obtained by computer simulation. The computer simulation was conducted wihtout the Gaussian assumption (see after equation (18)) made for the analytical performance analysis. It is seen that the difference between the analytical and simulation results is insignificant. Therefore, the analytical method used is a valid and fast technique to obtain the performance.

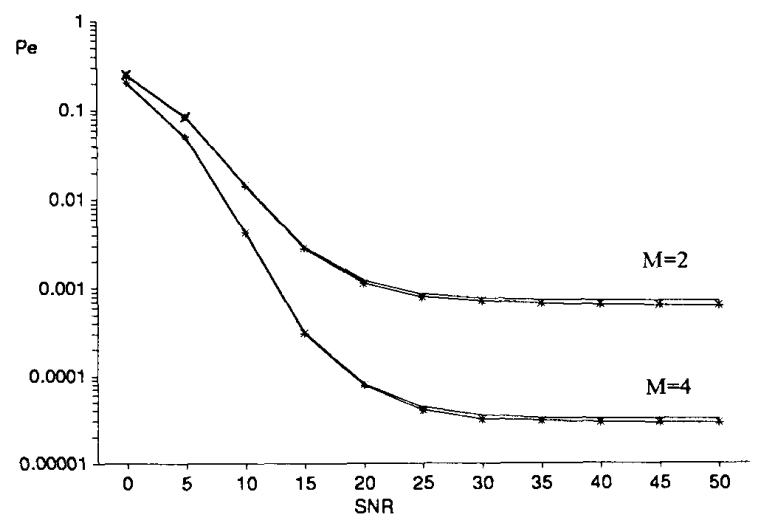

Fig. 7. Comparison of results obtained analytically (-) and results obtained by computer simulation $\left(-^{*} *_{-}\right)$. Results are shown for $\mathrm{R}=6.8$ $\mathrm{dB}, \mathrm{L}=4, \mathrm{~N}=255$, and $\mathrm{M}=2.4$.

2) Bit error probability with forward error correcting coding and diversity: In the plots for a system with FEC coding the signal energy $E_{b}$ stands for the transmitted energy per information bit. This means that $\mathrm{E}_{\mathrm{b}}$ for a system with $(15,7)$ $\mathrm{BCH}$ coding is $15 / 7$ times the energy transmitted per code symbol. 
We have calculated the coding results with $M=1$ for the following cases: a) $\mathrm{R}=6.8 \mathrm{~dB}, \mathrm{~L}=1$ and $\mathrm{N}=255, \mathrm{~b}) \mathrm{R}=$ $6.8 \mathrm{~dB}, \mathrm{~L}=5$ and $\mathrm{N}=255$. Besides the curves for coding without diversity, Figure 8 also contains the curves for selection diversity with $M=2,3$ and 4 .
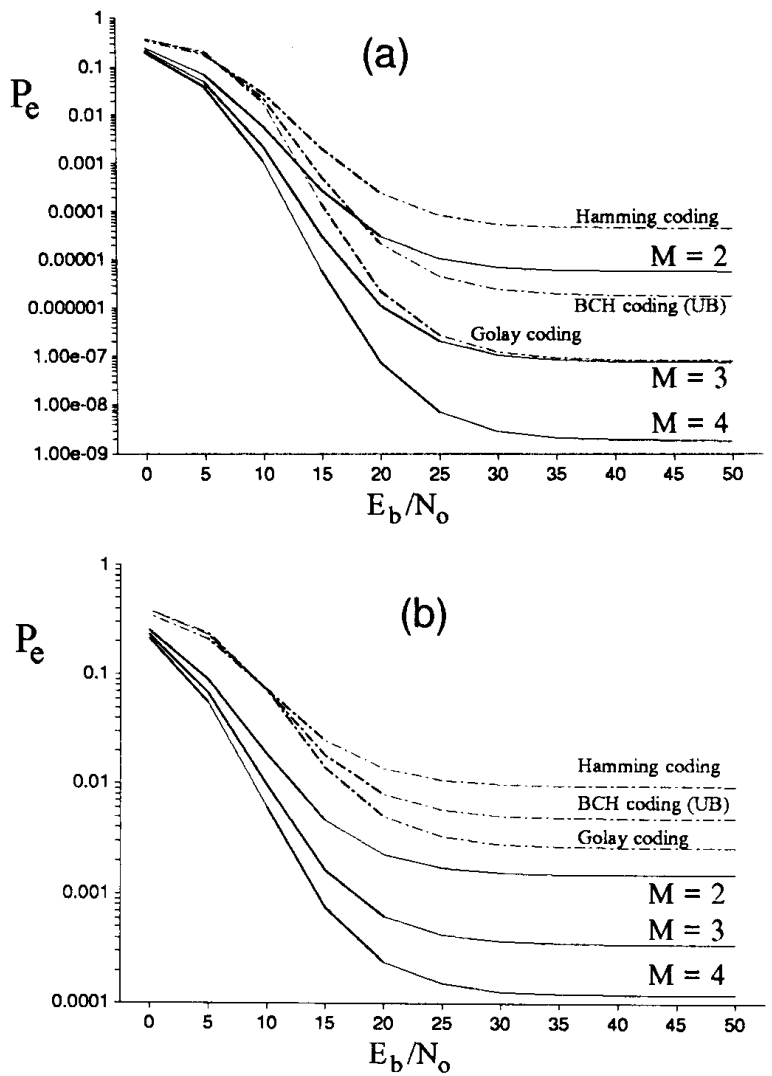

Fig. 8. Comparison of the bit error probability using FEC coding only (---) and selection diversity only (- $\longrightarrow$ for $R=6.8 \mathrm{~dB}, \mathrm{~N}=255$ and (a) $\mathrm{L}$ $=1$ and $(b) L=5$.

It is seen that for low values of $L$ (such as $L=1$ ) the use of FEC coding without diversity can lead to acceptable bit error probabilities. However, for larger values of $L(L=5)$ the use of FEC coding only is not sufficient. In that case diversity is necessary either in combination with FEC coding or not. Combining selection diversity with FEC coding is attractive when the bit error probability in the case of $\mathbf{M}=$ $\mathrm{L}$ (maximum order of diversity with one antenna) is not sufficiently low. Using FEC coding then avoids the need for installing additional antennas.

We have already seen that both longer spread spectrum codes and FEC codes improve the performance at the expense of an increased bandwidth. It is then interesting to compare the performance for $\mathrm{N}=127+$ FEC coding with the performance for $\mathrm{N}=255$ without coding (Figure 9). Since all three FEC codes approximately double the required signal bandwidth, the two cases mentioned above require approximately the same amount of bandwidth. It is seen that in the case of Golay coding the bandwidth efficiency of $\mathrm{N}=127+\mathrm{FEC}$ coding is higher since the performance is better than in the case of $\mathrm{N}=255$ without coding for equal bandwidth. Calculations have also shown that the performance is more sensitive to the (interference) noise level if FEC codes are used. This implies that if the number of users becomes too large, the use of spread spectrum codes of 255 chips will offer better performance than codes of 127 chips in combination with FEC codes.

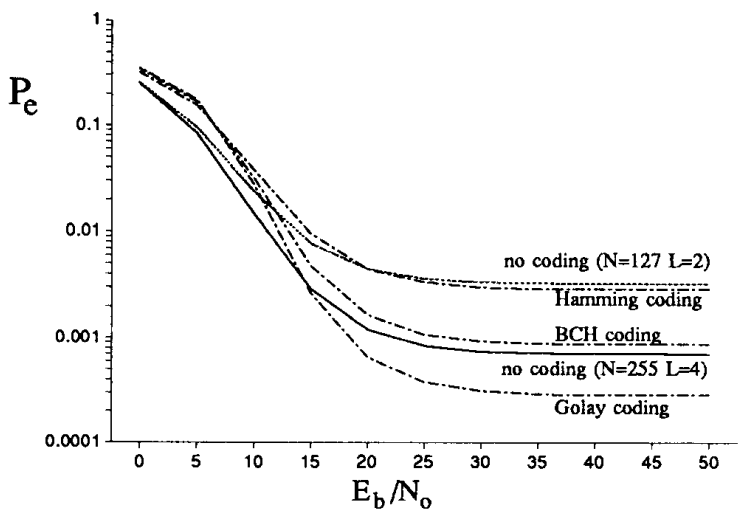

Fig. 9. Bit error probabilities with $r_{b}=64 \mathrm{kbit} / \mathrm{s}, T_{m}=150 \mathrm{~ns}, R=6.8$ $\mathrm{dB}$ and selection diversity with $\mathrm{M}=2$ for 1$) \mathrm{N}=127+\mathrm{FEC}$ coding and $\mathrm{L}=2$, and 2) $\mathrm{N}=255$ without $\mathrm{FEC}$ coding and $\mathrm{L}=4$.

3) Outage probability: We now consider the second measure of performance, the outage probability which is defined as the probability that the instantaneous bit error probability exceeds a certain threshold. Unless specified otherwise, the number of simultaneous transmitting users is $K=15$. Using equation (21) we computed the outage probability for selection diversity with $R=6.8 \mathrm{~dB}, \mathrm{~L}=5$ and $N=255$ for three values of the bit error threshold

TABLE I

The outage probability as a function of ber $_{o}$ and $\mathrm{E}_{\mathrm{b}} / \mathrm{N}_{\mathrm{o}}$ for selection diversity with $\mathrm{M}=1,4$ and $\mathrm{R}=6.8 \mathrm{~dB}, \mathrm{~L}=5$ and $\mathrm{N}=255$

\begin{tabular}{|c|c|c|c|c|}
\hline $\begin{array}{l}\mathrm{E}_{\mathrm{b}} / \mathrm{N}_{\mathrm{o}} \\
\mathrm{ber}_{\mathrm{o}}\end{array}$ & $10 \mathrm{~dB}$ & $20 \mathrm{~dB}$ & $30 \mathrm{~dB}$ & \\
\hline \multirow{2}{*}{$1 \mathrm{E}-1$} & $1.7 \mathrm{E}-1$ & $4.4 \mathrm{E}-2$ & $3.6 \mathrm{E}-2$ & $M=1$ \\
\hline & $7.7 \mathrm{E}-4$ & $3.8 \mathrm{E}-6$ & $1.6 \mathrm{E}-6$ & $M=4$ \\
\hline \multirow{2}{*}{$1 \mathrm{E}-2$} & $6.4 \mathrm{E}-1$ & 2.3E-1 & $1.9 \mathrm{E}-1$ & $M=1$ \\
\hline & $1.74 \mathrm{E}-1$ & $2.6 \mathrm{E}-3$ & $1.2 \mathrm{E}-3$ & $M=4$ \\
\hline \multirow{2}{*}{$1 \mathrm{E}-3$} & $9.1 \mathrm{E}-1$ & 4.7E-1 & $4.0 \mathrm{E}-1$ & $M=1$ \\
\hline & $6.8 \mathrm{E}-1$ & $4.9 \mathrm{E}-2$ & $2.6 \mathrm{E}-2$ & $M=4$ \\
\hline
\end{tabular}


(ber ${ }_{o}$ ) and three different values of the signal to noise ratio. This was done for $M=1$ (no diversity) and $M=4$. The results are shown in Table $I$.

In Figure 10 the effect of the delay spread is depicted for $\mathrm{N}=255, \mathrm{M}=4, \mathrm{R}=6.8 \mathrm{~dB}, \mathrm{~L}=1,5,10$ and ber $_{o}=10^{-2}$. As expected, the outage probability increases as the number of resolvable paths increases due to increase in delay spread for $\mathrm{N}=255$.

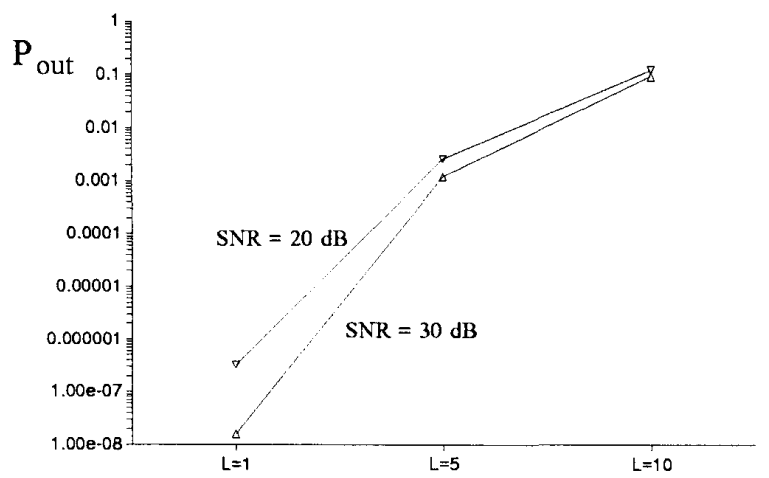

Fig. 10. The influence of the delay spread on the outage probability for $R$ $=6.8 \mathrm{~dB}$, selection diversity with $\mathrm{M}=4$ and $b e r_{o}=10^{-2}$.

Calculations confirm that maximal ratio combining yields better performance than selection diversity in terms of outage probability.

4) Bandwidth efficiency: Using equation (30) the bandwidth efficiency is evaluated for both selection diversity and maximal ratio combining. Unless specified otherwhise ber $_{b e}=10^{-4}$ and the signal-to-noise ratio $\mathrm{E}_{\mathrm{b}} / \mathrm{N}_{\mathrm{o}}=20 \mathrm{~dB}$.

In Table II the bandwidth efficiency is given for selection diversity with four different values of the number of resolvable paths $\mathrm{L}=1,2,4$ and 8 , and three orders of diversity $M=1,2$ and 4 . Gold codes of $N=255$ chips are used. There are no results shown for those cases where the error probability is always greater than ber $_{b e}=10^{-4}$. We see from Table II that an increase in the order of diversity gives an increase in the bandwidth efficiency, and that an increase in $\mathbf{L}$ gives a decrease in the bandwidth efficiency.

TABLE II

The bandwidth efficiency as a function of $\mathrm{M}$ and $\mathrm{L}$ for selection diversity with $\mathrm{N}=255, \mathrm{R}=6.8 \mathrm{~dB}$ and ber $_{b e}=10^{-4}$

\begin{tabular}{||c|c|c|c|c||}
\hline $\begin{array}{c}\mathrm{L} \\
\mathrm{M}\end{array}$ & $\mathrm{L}=1$ & $\mathrm{~L}=2$ & $\mathrm{~L}=4$ & $\mathrm{~L}=8$ \\
\hline 1 & - & - & - & \\
\hline 2 & 0.098 & 0.055 & 0.031 & 0.012 \\
\hline 4 & 0.214 & 0.090 & 0.051 & 0.031 \\
\hline
\end{tabular}

In Table III we make a comparison between the performance of Gold codes of 127 chips and codes of 255 chips with forward error correcting coding, for both selection and maximal ratio combining. We presume that the data rate, $r_{b}$, and the rms delay, $T_{m}$, are the same in both the cases. This means that the number of resolvable paths is doubled in the case of $\mathrm{N}=255\left(\mathrm{~L}_{255}\right)$ as compared to the case of $\mathrm{N}=127\left(\mathrm{~L}_{127}\right)$. Results are shown for $\mathrm{L}_{255}=2 \mathrm{~L}_{127}$ $=4$ for three orders of diversity $M=1,2$ and 4 . From Table III we see that $\mathrm{N}=127+$ FEC codes + diversity, offers

TABLE III

Bandwidth efficiency for selection diversity and maximal ratio combining with $\mathrm{FEC}$ coding for $\mathrm{N}=127,255$ and $\mathrm{M}=1,2,4$

\begin{tabular}{|c|c|c|c|c|c|c|}
\hline & \multicolumn{3}{|c|}{$N=127$} & \multicolumn{3}{|c|}{$\mathrm{N}=255$} \\
\hline & Hamming & $\mathrm{BCH}$ & Golay & Hamming & $\mathrm{BCH}$ & Golay \\
\hline$M=1$ & 0.004 & 0.015 & 0.021 & 0.011 & 0.016 & 0.025 \\
\hline$M=2$ & 0.027 & 0.040 & 0.066 & 0.025 & 0.027 & 0.035 \\
\hline$M=4$ & 0.040 & 0.055 & 0.082 & 0.031 & 0.033 & 0.041 \\
\hline \multicolumn{7}{|c|}{$\uparrow$ SELECTION DIVERSITY $\uparrow$} \\
\hline \multicolumn{7}{|c|}{$\downarrow$ MAXIMAL RATIO COMBINING $\downarrow$} \\
\hline$M=1$ & 0.004 & 0.015 & 0.021 & 0.11 & 0.016 & 0.025 \\
\hline $\mathbf{M}=2$ & 0.041 & 0.048 & 0.069 & 0.034 & 0.037 & 0.041 \\
\hline$M=4$ & 0.112 & 0.107 & 0.136 & 0.067 & 0.071 & 0.080 \\
\hline
\end{tabular}


superior performance in terms of bandwidth efficiency, as compared to $\mathrm{N}=255+$ FEC codes + diversity.

We now compare the case $\mathrm{N}=255, \mathrm{~L}=4$ with selection diversity (Table II) with the case $\mathrm{N}=127, \mathrm{~L}=2$ with FEC codes and diversity (Table III). These two cases require approximately the same amount of bandwidth. It is seen that $\mathrm{N}=127+\mathrm{FEC}$ codes is superior to $\mathrm{N}=255$ in terms of bandwidth efficiency.

\section{CoNCLUSIONS}

We have evaluated the performance of a star-connected DS/SS system for indoor wireless applications. The indoor radio channel was assumed to be of the Rician fading type, and DPSK modulation was used. Two types of diversity, selection diversity and maximal ratio combining were considered. The performance was assessed in terms of bit error probability, outage probability and bandwidth efficiency.

In general it can be stated that diversity improves the performance (bit error probability, outage probability, bandwidth efficiency) significantly, and that maximal ratio combining yields superior performance as compared to selection diversity.

It was seen that the performance is quite sensitive to the value of the rms delay spread and the bit rate at which data is transmitted. In general the performance is affected (worsens) if an increased value of the rms delay spread or bit rate causes the number of resolvable paths, $L$, to increase.

Methods to improve the performance for a given bit rate and rms delay spread are investigated using diversity (selection diversity and maximal ratio combining), FEC coding (Hamming codes, BCH codes, and Golay codes), and longer spread spectrum codes. These methods can also be combined. The drawback of diversity is that if it is necessary to have an order of diversity, $M$, larger than the number of resolvable paths, then the use of multiple antennas is required. The drawback of FEC codes and longer spread spectrum codes is that more bandwidth is required. In all cases additional hardware and logic are required for implementation of the system. From the results the following can be concluded.

1) Instead of installing multiple (two or more) antennas per receiver FEC coding can be used either independent or in combination with diversity to further improve the performance at the cost of an increased system bandwidth. The two options should be compared in terms of implementation complexity and costs.

2) FEC coding can not completely replace for diversity.
The performance with FEC coding deteriorates faster than the performance for diversity if the number of paths or simultaneously active users increases.

3) Using spread spectrum codes of 255 chips improve the bit error probability at the cost of a higher system bandwidth as compared to spread spectrum codes of 127 chips. However, the improvement of the bit error probability is not such that the bandwidth efficiency is improved.

4) If the interference power is below a certain limit, a system with FEC coding and spread spectrum codes of 127 chips can compete with a system with spread spectrum codes of 255 chips (note that these two system require the same amount of bandwidth). This holds for both bit error probability and bandwidth efficiency. If the number of paths is too large or the number of active users crosses a certain limit, the system with spread spectrum codes of 255 chips but without FEC codes, yields better performance.

\section{REFERENCES}

[1] M. Kavehrad, "Performance of Nondiversity Receivers for Spread Spectrum in Indoor Wireless Communication," $A T \& T$ Technical Journal, vol. 64, no. 6, pp. 1181-1210, July-August 1985.

[2] M. Kavehrad and P. J. McLane, "Performance of Low-Complexity Channel Coding and Diversity for Spread Spectrum in Indoor, Wireless Communication," $A T \& T$ Technical Journal, vol. 64, no. 8 , pp. 1927-1965, October 1985.

[3] M. Kavehrad and B. Ramamurthi, "Direct-Sequence Spread Spectrum with DPSK Modulation and Diversity for Indoor Wireless Communication," IEEE Transactions on Communications, vol. Com-35, no. 2, pp. 224-236, February 1987.

[4] M. Kavehrad and P. J. McLane, "Spread Spectrum for Indoor Digital Radio," IEEE Communications Magazine, vol. 25, no. 6, pp. 32-40, June 1987.

[5] M. Kavehrad and G.E. Bodeep, "Design and Experimental Results for a Direct-Sequence Spread-Spectrum Radio Using Differential Phase-Shift Keying Modulation for Indoor, Wireless Communications," IEEE Journal on Selected Areas in Communications, vol. SAC-5, no. 5, pp. 815-823, June 1987.

[6] K. Pahlavan, "Wireless Communication for Office Information Networks," IEEE Communications Magazine, vol. 23, no. 6, pp. 19-26, June 1985.

[7] K. Pahlavan and M. Chase, "Spread Spectrum Multiple-Access Performance of Orthogonal Codes for Indoor Radio Communications," IEEE Transactions on Communications, vol. 38 , no. 5, pp. 574-577, May 1990.

[8] R. Prasad, H.S. Misser, A. Kegel, "Performance Ananlysis of DirectSequence Spread-Spectrum Multiple Access communication in an Indoor Rician-Fading channel with DPSK modulation," Electronics Letters, vol. 26. no. 17, pp. 1366-1367, August 1990.

[9] R.J.C. Bultitude, "Measurement, Characterization and Modeling of Indoor $800 / 900 \mathrm{MHz}$ Radio Channels for Digital Communications," IEEE Communications Magazine, vol. 25, no. 6, pp. 5-12, June 1987.

[10] R.J.C. Bultitude, S.A. Mahmoud and W.A. Sullivan, "A comparison of indoor radio propagation characteristics at $910 \mathrm{MHz}$ and 1.75 $\mathrm{GHz}, "$ IEEE journal on selected areas in communications, vol. 7, no. 1, January 1989. 
[11] T.S. Rappaport and C.D. McGillem, "UHF Fading in Factories," IEEE Journal on Selected Areas in Communications, vol. 7, no. 1, pp. 40-48, January 1989.

[12] D.M.J. Devasirvatham, C. Banarjee, M.J. Krain and Rappaport, "Multi-Frequency Radiowave propagation Measurements in the Portable Radio Environment," Proceedings IEEE International Conference on Communications (ICC'90), Atlanta, vol. 4 of 4, pp. 335.1.1-7, April 1990.

[13] R. Prasad, "Throughput analysis of slotted code division multiple access for indoor radio channels," Proc. IEEE Symposium on spread spectrum techniques and applications, King's College, London, pp. 12-17, September 1990.

[14] A. Zigic and R. Prasad, "Computer simulation of indoor data channels with a linear adaptive equalizer," Electronic Letters, vol. 26, no. 19, pp. 1596-1597, September 1990.

[15] J.G. Proakis, "Digital Communications (second edition)," McGrawHill Book Company, New York, 1989.

[16] J. Wang and M. Moeneclaey, "Hybrid DS/SFH-SSMA with predetection diversity and coding over indoor radio multipath Rician-fading channels," IEEE Transactions on Communications, vol. COM-40, October, 1992.

Ramjee Prasad (M'88-SM'90) was born in Babhnaur (Gaya), Bihar, India on July 1, 1946. He received the B.Sc. (Eng.) from Bihar Institute of Technology, Sindri, India, and the M.Sc. (Eng.) and Ph.D. degrees from Birla Institute of Technology (BIT), Ranchi, India in 1968, 1970 and 1979 , respectively.

He joined BIT as Senior Research Fellow in 1970 and became Associate Professor in 1980. During 1983-1988 he was with the University of Dar es Salaam (UDSM), Tanzania, where he became Professor in Telecommunications at the Department of Electrical Engineering 1986. Since February 1988, he has been with the Telecommunications and Traffic Control Systems Group, Delft University of Technology, The Netherlands, where he is actively involved in the area of mobile, indoor and personal radio communications. While he was with BIT, he supervised many research projects in the area of Microwave and Plasma Engineering. At UDSM he was responsible for the collaborative project "Satellite Communications for Rural Zones" with Eindhoven University of Technology, The Netherlands. He has published over 200 technical papers. His current research interest lies in packet communications, adaptive equalizers, spread-spectrum CDMA systems and multi-media communications.

He has served as a member of advisory and programme committees of several IEEE international conferences. He has also presented tutorials on Mobile and Indoor Radio Communications at various universities, technical institutions and IEEE conferences. He is also a member of a working group of European co-operation in the field of scientific and technical research (COST-231) project dealing with "Evolution of Land Mobile Radio (including personal) Communications" as an expert for the Netherlands.

Prof. Prasad is listed in the US Who's Who in the World. He was Organizer and Interim Chairman of IEEE Vehicular Technology/Communications Society Joint Chapter, Benelux Section. Now he is the elected chairman of the joint chapter. He is also founder of the IEEE Symposium on Communications and Vehicular Technology (SCVT) in the Benelux and he was the Symposium Chairman of SCVT'93. He is the Co-ordinating Editors and one of the Editors-inChief of a Kluwer international journal on "Wireless Personal Communications" and also a member of the editorial board of other international journals including IEEE Communications Magazine. H was the Technical Program Chairman of PIMRC'94 International Symposium held in The Hague, The Netherlands during September 1923, 1994 and also of the Third Communication Theory Mini-Conference in conjunction with GLOBECOM'94 held in San Francisco, California during November 27-30, 1994. He is a Fellow of IEE, a Fellow of the Institution of Electronics \& Telecommunication Engineers, a Senior Member of IEEE and a Member of the New York Academy of Sciences and of NERG (The Netherlands Electronics and Radio Society).
Howard S. Misser (S'89-M'91) was born in Paramaribo, Surinam, on May 11, 1968. He received the M.Sc. degree in Electrical Engineering from Delft University, Delft, The Netherlands, in 1990.

He worked at the Telecommunications and Traffic-Control Systems Group of the same university as a Research Fellow. He is currently with the Dr. Neher Laboratories of the Royal Dutch PTT in Leidschendam, The Netherlands. His research interests are in the fields of broadband network technologies and architecture, radio and mobile communications, and spread-spectrum communication.

Adriaan Kegel was born in The Netherlands on November 4, 1932. He received the M.Sc. degree from Delft University of Technology, The Netherlands, in 1972. He joined Philips in 1954 where he worked on the development of microwave link equipment as a Radio Engineer. In 1964 he joined Delft University of Technology where he became an Associate Professor in 1985. In 1970 he became Chairman of the "Working Group Indonesia", which developed a low cost educational broadcasting system. For this development the working group received an award from the Scientific Radio Foundation Veder. After completion of the Indonesian Project in 1974, he continued his research work on the coding and transmission of graphical information. This work led to the development of several systems (e.g. Vidibord) and a number of publications (e.g. on Differential Chain Coding). He took retirement from his active service in the field of telecommunications in December 1993. His current interest is in photography and nature.

Mr. Kegel is a member of NERG (the Netherlands Electronics and Radio Society), a member of the FITCE (Federation of Telecommunication Engineers of the European Community) and a member of IEEE. 